5. Shibata S, Miyaji T, Iuchi I, Oba Y, Yamamoto K. Amino acid substitution in hemoglobin Makita. J Biochem 1968;63:193-8.

6. Shibata S, Yawata Y, Yamada O, Koresawa S, Ueda S. Altered erythropoiesis and increased hemolysis in hemoglobin M Akita (M Hyde Park beta92 His replaced by Tyr) disease. Hemoglobin 1976;1:111-24

7. Hutt PJ, Pisciotta AV, Fairbanks VF, Thibodeau SN, Green MM. DNA sequence analysis proves $\mathrm{Hb}$ M-Milwaukee- 2 is due to beta-globin gene codon 92 (CAC-- > TAC), the presumed mutation of Hb M-Hyde Park and Hb M-Akita. Hemoglobin 1998;22:1-10.

8. Stamatoyannopoulos G, Nute PE, Giblett E, Detter J, Chard R. Haemoglobin M Hyde Park occurring as a fresh mutation: diagnostic, structural, and genetic considerations. J Med Genet 1976;13:142-7.

9. Rotoli B, Camera A, Fontana R, et al. Hb-M “Hyde Park": a de novo mutation, identified by mass spectrometry and DNA analysis. Haematologica 1992;77:110-8.

10. Kumar GV, Sharma P, Chhabra S, Hira JK, Trehan A, Das R. Hb M-Iwate in an Indian family. Clin Chim Acta 2015;446:192-4.

11. Kedar PS, Nadkarni AH, Phanasgoankar S, et al. Congenital methemoglobinemia caused by Hb-MRatnagiri (beta-63CAT-- > TAT, His-- > Tyr) in an Indian family. Am J Hematol 2005;79:168-70.

12. Upadhye D, Koduri P, Tarakeshwari S, et al. Hb M Hyde Park and $\mathrm{Hb} \mathrm{M}$ Boston in two Indian families - a rare cause of methaemoglobinemia. Int J Lab Hematol 2015;37:e40-3.

13. Loong TY, Chong DLS, Jamal ARA, Murad NAA, Sabudin RZAR, Fun LC. First reported case of haemoglobin-M Hyde Park in a Malay family living in Malaysia. EXCLI J 2016;15:630-5.

14. Stucke AG, Riess ML, Connolly LA. Hemoglobin M (Milwaukee) affects arterial oxygen saturation and makes pulse oximetry unreliable. Anesthesiology 2006;104:887-8.

\section{Nuances in the management of acquired hemophilia $A$ in an elderly patient with large granular lymphocytic leukemia}

TO THE EDITOR: Acquired hemophilia A (AHA) is a rare (1-4 cases/million/yr) often underdiagnosed bleeding diathesis caused by polyclonal neutralizing immunoglobulins (IgG1 and IgG4) that block the interaction of factor VIII (FVIII) with factor IX, phospholipids and von Willebrand factor. Although it could be idiopathic, it has been described in association with various autoimmune disorders, pregnancy, blood and solid organ malignancies, and certain drugs. Lack of clear treatment guidelines and poor prognosis (mortality, 8-22\%) makes AHA an object of concern for clinicians [1]. We discuss the case of a patient with large granular lymphocytic leukemia (LGLL) who presented with acute onset of bleeding and was diagnosed with AHA. To our knowledge, this association has only been reported once in the literature [2]. The nuances in managing AHA with the available hemostatic products and immunosuppressive therapies are also explored.

An 82-year-old man diagnosed with LGLL two years earlier for which he was being observed, presented with extensive non-traumatic bruising of both hips and the left side of the chest wall. His hemoglobin on admission was $9.9 \mathrm{~g} / \mathrm{dL}$ which progressively decreased to $7.4 \mathrm{~g} / \mathrm{dL}$. The platelet count $(184,000 / \mu \mathrm{L})$ and absolute neutrophil count $\left(5.5 \times 10^{9} / \mathrm{L}\right)$ were normal. Hemoglobin level briskly fell to $6.5 \mathrm{~g} / \mathrm{dL}$ after a single bout of rectal bleed despite performing one-unit blood transfusion. The patient had a history of multiple vascular comorbidities, such as coronary artery disease, bilateral iliac artery occlusions, and left partial carotid artery occlusion, which were corrected by percutaneous cutaneous intervention, aortoiliac vascular graft, and endarterectomy, respectively. Aspirin and clopidogrel started for these vascular comorbidities were discontinued. The activated partial thromboplastin time (aPTT) was elevated (80 s) and was not corrected on 1:1 mixing study with normal plasma. The prothrombin time was normal while circulating FVIII inhibitor level was 15 Bethesda Units (BU), suggestive of AHA. Lupus anticoagulant, Coombs test, and disseminated intravascular coagulation workup were negative; however, FVIII levels were found to be markedly reduced (less than $2 \%)$ leading to a diagnosis of AHA. Due to the patient's age and vascular comorbidities, the use of bypassing agents such as rFVII or FVIII inhibitor bypass activity for hemostasis was avoided as there were concerns for thrombotic risk. Initially, the patient was administered 6,000 units of porcine FVIII to achieve $30 \%$ of plasma FVIII activity. Further, the dose was titrated to 200 units/kg to achieve $50 \%$ activity. He was simultaneously treated with immunosuppressive therapy using prednisone $(1 \mathrm{mg} / \mathrm{kg} / \mathrm{d})$, cyclophosphamide (100 mg/d), and rituximab $\left(375 \mathrm{mg} / \mathrm{m}^{2} / \mathrm{wk}\right.$ for $4 \mathrm{wk}$ ) to reverse the inhibitor. When he visited our outpatient clinic for the last dose of rituximab, FVIII was up to $19 \%$ and FVIII inhibitor had reduced to 4 BU (down from $15 \mathrm{BU}$ ). He did not have any clinically overt bleeding at that time, suggesting partial remission.

AHA typically presents with acute onset of bleeding without any prior history of coagulopathy in a bimodal age distribution of 20-30 years and 60-80 years, respectively. Nearly half of the cases are idiopathic while the rest are associated with autoimmune conditions, malignancies, and drugs. It has also been described in pregnancy, particularly in the postpartum setting. Clinically, $80 \%$ of cases with FVIII antibodies present with skin, soft tissue, and mucosal bleeds. This is different from congenital FVIII deficiency which typically presents with hemarthroses [1]. Although the spectrum of bleeding in AHA ranges from minor superficial to gastrointestinal, urological bleeding, and retroperitoneal hematomas, in $90 \%$ of cases, it strongly tends towards the latter life-threatening type of bleeding [1]. AHA diagnosis requires a sound history corroborated with lab findings, a prolonged aPTT not corrected on mixing with normal plasma, FVIII assay, and a circulating inhibitor of FVIII [3, 4]. 
LGLL is a lymphoproliferative disorder that is known to be associated with autoimmune conditions, such as rheumatoid arthritis and Sjogren's syndrome. To our knowledge, it has only been reported once in association with AHA. This case would be the second of such report of LGLL as a potential cause of AHA [2]. While our predecessors managed bleeding using bypassing agents, our choice varied based on the patient's age and comorbidities [2].

Hemostasis in AHA can be achieved by administering FVIII bypassing agents in the form of recombinant activated factor VII (rFVIIa) or activated prothrombin complex concentrate (aPCC) which can generate thrombin in a non-physiologic fashion, independent of FVIII. A practical limitation of these products is that their activity cannot be monitored with standard coagulation assays and they carry an increased risk of thrombosis, particularly in older individuals with vascular co-morbidities. Recombinant porcine FVIII (rPorcine FVIII) is an alternative hemostatic agent for AHA since it is quite different from human FVIII and is not recognized by the autoantibody in most cases, thus allowing the coagulation cascade to proceed in a normal physiologic fashion. An advantage of this is that it can be measured by checking FVIII activity, thereby allowing it to be measured to maintain an activity that minimizes the risk of both bleeding due to sub-therapeutic levels and thrombosis from supra-therapeutic levels. Due to this, we prefer rPorcine FVIII over bypassing agents especially in older patients with cardiovascular comorbidities [3]. While there are no head-to-head comparison studies between the two, the decision is usually based on the availability, prior case-to-case experience, and economic considerations.

Immunosuppressive therapy (IST) is recommended in all adults with AHA to achieve FVIII inhibitor eradication as the bleeding-related morbidity and mortality is substantial. In multiple studies and meta-analyses, it was found that combination IST (corticosteroids, cyclophosphamide, and rituximab) was superior to corticosteroids alone [3].

Our patient had LGLL as a potential underlying trigger for the development of AHA. Usually, this is an indolent neoplasm not requiring specific management other than watchful observation. However, concurrence with AHA and the patient's age necessitated chemotherapy with cyclophosphamide. Other instances where LGLL requires treatment are severe neutropenia (absolute neutrophil count or ANC $<0.5 \times 10^{9} / \mathrm{L}$ ), moderate neutropenia (ANC $>0.5 \times 10^{9} / \mathrm{L}$ ) with superinfections, autoimmune conditions needing treatment, and transfusion-dependent anemia [5].

In summary, our case is the second report in the literature on AHA occurring in association with LGLL. Also, the discussion of our therapeutic choice for hemostasis presents nuances in the management of AHA in the elderly and those with cardiovascular comorbidities.
Nishant R. Tiwari ${ }^{1}$, Kaustubh S. Chaudhari ${ }^{2}$, Vrushab Rao ${ }^{3}$, Vivek R. Sharma ${ }^{4}$

Department of Internal Medicine, ${ }^{1}$ Byramjee Jeejeebhoy Medical College, 'Jagtap Clinic and Research Center, Pune,

${ }^{3}$ Bangalore Medical College and Research Institute,

Bangalore, India, 'James Graham Brown Cancer Center, University of Louisville, Louisville, KY, USA

Correspondence to: Nishant R. Tiwari

Department of Internal Medicine, Byramjee Jeejeebhoy Medical College, Pune 411001, India

E-mail: dr.nishant.tiwari.1996@gmail.com

Received on Apr. 24, 2020; Revised on May 15, 2020; Accepted on Jul. 24, 2020 https://doi.org/10.5045/br.2020.2020093

\section{Authors' Disclosures of Potential Conflicts of Interest}

No potential conflicts of interest relevant to this article were reported.

\section{REFERENCES}

1. Franchini M, Lippi G. Acquired factor VIII inhibitors. Blood 2008;112:250-5.

2. Murphy PW, Brett LK, Verla-Tebit E, Macik BG, Loughran TP Jr. Acquired inhibitors to factor VIII and fibrinogen in the setting of T-cell large granular lymphocyte leukemia: a case report and review of the literature. Blood Coagul Fibrinolysis 2015;26: 211-3.

3. Kruse-Jarres R, Kempton CL, Baudo F, et al. Acquired hemophilia A: updated review of evidence and treatment guidance. Am J Hematol 2017;92:695-705.

4. Delgado J, Jimenez-Yuste V, Hernandez-Navarro F, Villar A. Acquired haemophilia: review and meta-analysis focused on therapy and prognostic factors. Br J Haematol 2003;121:21-35.

5. Lamy T, Moignet A, Loughran TP Jr. LGL leukemia: from pathogenesis to treatment. Blood 2017;129:1082-94.

\section{An illustrative case of B-cell prolymphocytic leukemia}

TO THE EDITOR: B-cell prolymphocytic leukemia (B-PLL) is an extremely rare malignancy ( $<1 \%$ of B-cell leukemias) $[1,2]$. Given a wide array of differential diagnoses, establishing the correct diagnosis is challenging [3]. Histomorphological evaluation of the peripheral blood (PB) smear and diligent interpretation of the immunophenotype of lymphocytes by an expert pathologist are essential for the diagnosis of B-PLL. In this report, we describe the case of a patient with B-PLL, discuss the diagnostic possibilities, and briefly review the relevant literature.

A 65-year-old man presented to our hospital in August 2019 with the complaints of fever and left upper abdomen pain for 6 months. General examination showed pallor. 